\title{
Pancreatic Mucinous-Cystic Neoplasm with Low Grade Dysplasia
}

National Cancer Institute

\section{Source}

National Cancer Institute. Pancreatic Mucinous-Cystic Neoplasm with Low Grade

Dysplasia. NCI Thesaurus. Code C95483.

A non-invasive mucinous cystic neoplasm that arises from the pancreas and is

characterized by the presence of mild dysplasia and absence of mitotic figures. 